\title{
The prevalence of high risk of obstructive sleep apnea in patients with allergic rhinitis
}

\author{
Surintorn Wongvilairat, ${ }^{1}$ Paraya Assanasen, ${ }^{1}$ Wish Banhiran, ${ }^{1}$ Pongsakorn Tantilipikorn, ${ }^{1}$ Chaweewan Bunnag ${ }^{1}$
}

\section{Abstract}

Background: Although allergic rhinitis (AR) has not been acknowledged as a strong risk factor for obstructive sleep apnea (OSA), several pathophysiological linkages between these two conditions have frequently been reported. However, epidemiological data relating to the prevalence of OSA in patients with AR remain scarce.

Objective: To investigate the prevalence of patients at high risk for OSA among patients with AR, and to determine the relationship between OSA and severity of AR.

Methods: Patients aged $\geq 18$ years with a diagnosis of $\mathrm{AR}$ and positive skin prick test were recruited from the allergy clinic, Department of Otorhinolaryngology, Faculty of Medicine Siriraj Hospital, Mahidol University, Thailand between October 2014 to November 2015. During routine follow-up, participants were asked to complete the STOPBang questionnaire and visual analog scale regarding AR symptoms, including their duration and severity. Patients with STOP-Bang score of $\geq 3$ were considered as high risk for having OSA.

Results: Of the 120 AR patients (45 males, 75 females) included, there were 28 patients (19 males, 9 females) who had a STOP-Bang score of $\geq 3$ (23.3\%), especially for those who had longer duration of AR symptoms (low-risk/high-risk: $60 / 120$ months, $p=0.01$ ). However, no significant relationship was observed between ARIA classification and severity of nasal symptoms of AR for being high risk of OSA.

Conclusion: The prevalence of high risk of having OSA in patients with AR was two times higher than that in general Thai population. Early screening and further management for these patients may improve treatment outcomes and quality of life.

Key words: Obstructive sleep apnea, allergic rhinitis, snoring, STOP-Bang, Thai

\section{Citation:}

Wongvilairat, S., Assanasen, P., Banhiran, W., Tantilipikorn, P., Bunnag, C. (2022). The prevalence of high risk of obstructive sleep apnea in patients with allergic rhinitis. Asian Pac J Allergy Immunol, 40(3), 205-209. https://doi.org/10.12932/ap-1412180458

\section{Affiliation: \\ ${ }^{1}$ Department of Otorhinolaryngology, Faculty of Medicine Siriraj Hospital, Mahidol University, Bangkok, Thailand}

Corresponding author:

Wish Banhiran

Department of Otorhinolaryngology,

Faculty of Medicine Siriraj Hospital, Mahidol University

2 Wanglang Road, Bangkoknoi, Bangkok 10700, Thailand

E-mail:wishbanh@gmail.com,wishbanh@hotmail.com

\section{Introduction}

Allergic rhinitis (AR) is a highly prevalent immunological disease that often causes nasal obstruction that associates with poor sleep quality and obstructive sleep apnea (OSA). ${ }^{1-7}$ OSA is defined as an abnormal condition of upper airway collapse during sleep. If left untreated, especially in severe cases, OSA can lead to several adverse health consequences, including cardiovascular diseases and even death. ${ }^{8}$ Although AR has not been acknowledged as a strong risk factor for OSA, pathophysiological linkages (primarily relating to nasal obstruction) between these two common conditions have been reported., ${ }^{5-16}$ These linkages are often explained by mechanisms that include (1) an increase in upper airway collapsibility due to greater negative intraluminal pressure according to the theory of Starling resister model, which considers an oropharyngeal airway as a hollow collapsible tube lying between upstream and downstream fixed structures; (2) compensatory unstable oral breathing that leads to 
higher airway resistance and more narrowing of the pharyngeal lumen; (3) reduction in spontaneous ventilation due to the deactivation of nasal-ventilator reflex that is caused by bypassing of the nasal airway; and, (4) reduction in nitric oxide that results in perfusion ventilation mismatch. ${ }^{5,9}$ However, epidemiological data relating to the coexistence of and relationship between OSA and AR are still unclear.

Among OSA patients, the prevalence of $\mathrm{AR}$ is a high $35.2 \%$ according to the results of a meta-analysis conducted by Cao, et al. ${ }^{12}$ Our group previously reported a prevalence that was even higher among patients with symptoms of chronic rhinitis and suspected OSA. ${ }^{17}$ Other studies reported a prevalence of OSA of at least $10 \%$, and that it is more common in males than in females in general population. ${ }^{6,7,18}$ However, in patients with AR, the prevalence of OSA is unclear, although one study reported a prevalence rate of approximately $36 \% .{ }^{14}$ This scarcity of data may be due to the limitations of OSA diagnosis in general practice. Despite the fact that polysomnography (PSG), which is a comprehensive technician-attended sleep test, is considered a gold standard for the diagnosis of OSA, its disadvantages, including high cost, long waiting list, and laborious procedural features, often limits access to PSG for many patients. It is, thus, more practical to reserve PSG as a diagnostic method for those who are at high risk for being OSA. Therefore, it is usually screened by a much more simple questionnaire such as STOP-Bang. ${ }^{19,20}$ The aims of this study were to investigate the prevalence of patients at high risk for OSA among patients with $\mathrm{AR}$, and to determine the relationship between OSA and the severity of AR.

\section{Methods}

This cross-sectional study was conducted during October 2014 to November 2015 at the Department of Otorhinolaryngology, Faculty of Medicine Siriraj Hospital, Mahidol University after receiving approval from the Siriraj Institutional Review Board (SIRB) (COA no. Si506/2014). All included subjects provided written informed consent prior to participation in the study. This study complied with all of the principles set forth in the Declaration of Helsinki (1964) and all of its subsequent amendments.

\section{Subjects}

Patients satisfying all of the following criteria were included: age greater than or equal to 18 years, clinical diagnosis of AR based on the presence of symptoms that included sneezing, runny nose, itching, and/or nasal obstruction, and positive skin prick tests (the largest diameter of the reactive wheal was 3 millimeters or much larger than negative control). Patients unable or unwilling to answer the questionnaires were excluded. All participants were asked to answer all of the following elicitations: STOP-Bang questionnaire, questions relating to the severity and duration of AR symptoms (any of those previously mentioned) according to the Allergic Rhinitis and its Impact on Asthma (ARIA) guideline, and visual analog scale (VAS: 0-10) of nasal symptom severity.

\section{STOP-Bang questionnaire}

The STOP-Bang questionnaire is an 8-item yes/no questionnaire to determine risk of OSA that was developed by Chung, et al. ${ }^{19-22}$ The questionnaire contains subjective and objective questions, include those relating to snoring, tiredness, observed apnea, blood pressure, body mass index (BMI), age, neck circumference (NC), and gender. If patients answer yes to three or more items, they are considered to be at high-risk for having OSA. In this study, we used our Thai version of the STOP-Bang questionnaire, ${ }^{23}$ which was translated with permission. The Thai version showed sensitivity of $87.3 \%$, specificity of $48.1 \%$, positive predictive value (PPV) of $82.2 \%$, and negative predictive value (NPV) of $52.2 \%$ for the diagnosis of OSA compared to PSG in Thai patients.

\section{Questionnaires relating to AR symptoms}

According to the ARIA guideline ${ }^{24}$ patients with AR can be classified by their symptoms into 4 groups: (1) Mild intermittent group is defined as those who have nasal symptoms less than 4 days per week OR symptoms that persist for less than 4 weeks, but the symptoms do not adversely affect the quality of life; (2) Moderate to severe intermittent group is defined as those who have nasal symptoms less than 4 days per week OR symptoms that persist for less than 4 weeks, and the symptoms do adversely affect quality of life; (3) Mild persistent is defined as those who have nasal symptoms more than 4 days per week AND that persist for more than 4 weeks, but the symptoms do not adversely affect quality of life; and, (4) Moderate to severe persistent is defined as those who have nasal symptoms more than 4 days per week AND that persist for more than 4 weeks, and they do adversely affect quality of life.

The VAS questionnaire was used to evaluate the severity of each relevant nasal symptom before initial treatment (i.e., itching, sneezing, nasal obstruction, and runny nose). Scoring for each symptom ranged from 0 to 10 , with a higher score representing greater severity. According to Bousquet, et al. ${ }^{25}$ patients with AR could be classified by VAS into two groups: (1) mild AR for those who have a VAS score of less than 5; and, (2) moderate to severe AR for those who have a VAS score of 5 or more.

\section{Statistical analysis}

Continuous data are presented as mean \pm standard deviation (SD), and categorial data are shown as frequency and percentage. Independent t-test and Mann-Whitney $U$ test were used to compare continuous data between groups depending on the pattern of data distribution (parametric vs. non-parametric). Chi-square test for trend was used to analyze the relationship between the severity of disease and the risk of OSA. Statistical significance was set at a $p$-value less than 0.05. All data analyses were performed using SPSS Statistics v.22 (SPSS, Inc., Chicago, IL, USA). 


\section{Results}

A total of 120 patients (45 males and 75 females) with an age range of 18 to 65 years (mean: 34.2 ) and a mean BMI of $23.4 \mathrm{~kg} / \mathrm{m}^{2}$ (range: 16.4-40.2) were recruited. The mean neck circumference (NC) was $34.8 \mathrm{~cm}$ (range: 28-46). Only 4 patients had a BMI over $35 \mathrm{~kg} / \mathrm{m}^{2}$. The demographic data of patients comparing low risk and high risk group for OSA were shown in Table 1. The distribution of STOP-Bang scores among all participants is shown in Figure 1. There were 28 patients, 19 males and 9 females, who had STOP-Bang scores (number of items answered as "YES") of at least 3. Therefore, the overall prevalence of high risk for OSA among AR patients in this study was $23.3 \%$. Subgroup analysis of these patients showed a significantly higher prevalence of OSA in males (42.2\%) than females $(12.0 \%)(p=0.02)$.

The majority of patients $(50.0 \%)$ in this study were classified according to ARIA guideline as moderate to severe persistent AR (Table 2), with an observed non-significant increased likelihood of being high risk of OSA compared to mild AR. However, there was no significant difference between the low-risk group and the high-risk group among AR patients classified by this guideline $(p=0.60)$. Similarly, the severity of nasal symptoms (itching, sneezing, nasal obstruction, and runny nose) assessed only by VAS (0-10), was not significantly different between the low-risk and high-risk OSA groups (Table 3) ( $p>0.05$ ). This was corresponded with the results between the two groups proposed by Bousquet, et al. ${ }^{25}$ (mild AR vs. moderate to severe AR) and the low-risk and high-risk OSA groups $(p>0.05)$.

The duration of AR symptoms before treatment in the low risk for OSA group ranged from 1 to 384 months (median: 60 ), with an interquartile range of 24 and 120 months. The duration of symptoms in the high-risk group ranged from 12 to 360 months (median: 120), with an interquartile range of 60 and 138 months. The duration between groups was found to be significantly shorter in the low-risk group than in the high-risk group (Table 1) $(p=0.01)$.
Table 1. Demographic data of patients with low risk and high risk for OSA

\begin{tabular}{|l|c|c|c|}
\multicolumn{1}{|c|}{ Demographic data } & $\begin{array}{c}\text { Low risk of } \\
\text { OSA } \\
(\mathbf{N}=\mathbf{9 2})\end{array}$ & $\begin{array}{c}\text { High risk of } \\
\text { OSA } \\
(\mathbf{N}=\mathbf{2 8})\end{array}$ & p-value \\
\hline Gender (Male/Female) & $26 / 66$ & $19 / 9$ & 0.02 \\
Age (years) & $32.6 \pm 11.0$ & $39.4 \pm 11.9$ & 0.01 \\
Body mass index $\left(\mathrm{kg} / \mathrm{m}^{2}\right)$ & $22.8 \pm 3.7$ & $27.0 \pm 4.9$ & $<0.01$ \\
Neck circumference $(\mathrm{cm})$ & $33.7 \pm 2.8$ & $38.3 \pm 3.8$ & $<0.01$ \\
$\begin{array}{l}\text { Duration of rhinitis } \\
\text { (months) }\end{array}$ & $60(24,120)$ & $120(60,138)$ & 0.01 \\
\hline
\end{tabular}

A $p$-value of $<0.05$ indicates statistical significance.

The parametric continuous data were presented in mean \pm standard deviation. The non-parametric data (duration of rhinitis) were presented in median and interquartile range (percentile $25^{\text {th }}$, percentile $75^{\text {th }}$ ).

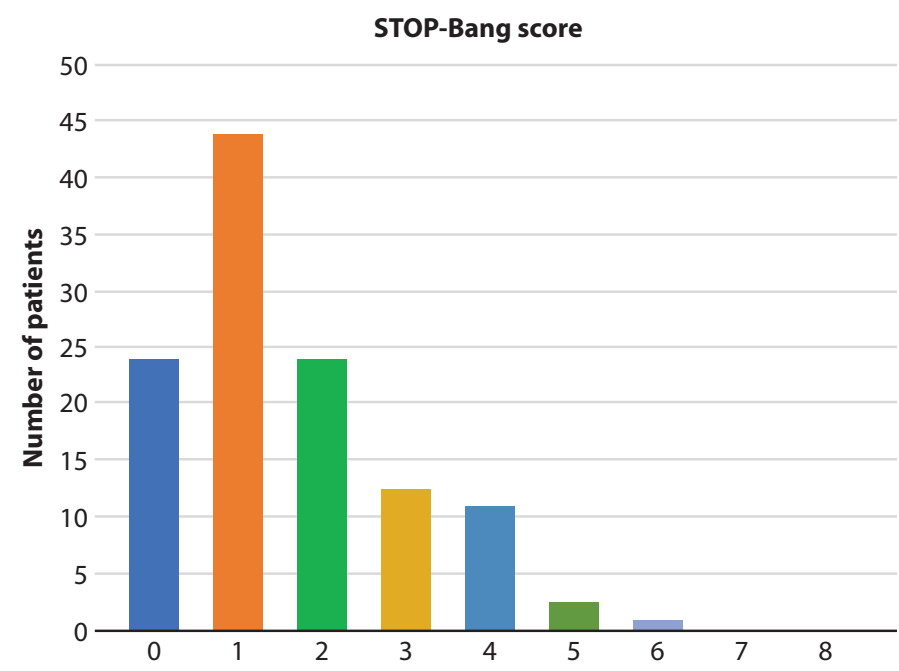

Figure 1. Distribution of STOP-Bang score

Table 2. Severity of allergic rhinitis and OSA risk

\begin{tabular}{|l|c|c|c|c|}
\multicolumn{1}{|c|}{ ARIA Classification } & $\begin{array}{c}\text { Number of } \\
\text { patients }\end{array}$ & $\begin{array}{c}\text { Low risk of OSA } \\
\text { N (\%) }\end{array}$ & $\begin{array}{c}\text { High risk of OSA } \\
\text { N (\%) }\end{array}$ & $p$-value \\
\hline 1. Mild intermittent & 25 & $20(80.0)$ & $5(20.0)$ & 0.66 \\
\hline 2. Mild persistent & 15 & $12(80.0)$ & $3(20.0)$ & 0.74 \\
\hline 3. Moderate to severe intermittent & 20 & $13(65.0)$ & $7(35.0)$ & 0.18 \\
\hline 4. Moderate to severe persistent & 60 & $47(78.3)$ & $13(21.7)$ & 0.67 \\
\hline
\end{tabular}

A $p$-value of $<0.05$ indicates statistical significance; Pearson's chi-square test: overall $p=0.60$

Abbreviations: OSA, obstructive sleep apnea; ARIA, Allergic Rhinitis and Its Impact on Asthma 
Table 3. Severity of nasal symptoms as assessed by visual analog scale compared between low and high risk of OSA

\begin{tabular}{|l|c|c|c|c|}
\hline \multicolumn{1}{|c|}{ Nasal symptoms } & VAS & Low risk of OSA & High risk of OSA & $p$-value \\
\hline Itching & $4.4 \pm 2.9$ & $4.3 \pm 2.9$ & $4.8 \pm 2.9$ & 0.50 \\
Sneezing & $4.3 \pm 2.8$ & $4.3 \pm 2.9$ & $4.2 \pm 2.5$ & 0.79 \\
Nasal obstruction & $5.6 \pm 2.7$ & $5.4 \pm 2.9$ & $6.1 \pm 2.0$ & 0.24 \\
Runny nose & $4.8 \pm 2.8$ & $5.1 \pm 2.8$ & $3.9 \pm 2.5$ & 0.06 \\
\hline
\end{tabular}

A $p$-value of $<0.05$ indicates statistical significance. The continuous data were presented in mean \pm standard deviation.

Abbreviations: OSA, obstructive sleep apnea; VAS, visual analog scale

\section{Discussion}

$\mathrm{AR}$ and OSA are common upper airway disorders that significantly affect patients worldwide, and that may lead to serious health consequences. ${ }^{1-8}$ Early detection and treatment of these conditions is the best way to improve patient quality of life and to prevent subsequent adverse side effects. Previous study showed that AR and OSA have some linkages, particularly relative to effects from nasal obstruction, ${ }^{9-11}$ and approximately one-third of OSA patients also had AR. ${ }^{3,12,17,26}$ Young, et al. ${ }^{27}$ reported that people with nasal obstruction due to allergy were 1.8 times more likely to have moderate to severe OSA. Stuck, et al. ${ }^{28}$ reported that seasonal AR could increase the mean apnea-hypopnea index (AHI) from 0.32 to 0.81 events/hr. However, only a few studies have focused on the prevalence of OSA in $\mathrm{AR}^{14,29}$ and data in South-East Asians has not yet been reported. Our findings revealed that $23.3 \%$ of AR patients are at high risk for OSA. This rate is approximately twice compared to the known prevalence of high risk for OSA in general Thai population. ${ }^{7}$ The results from the present study are lower than those reported by Kalpalklioglu, et al..$^{14}$ and Bozkurt, et al. ${ }^{29}$ who reported that $36 \%$ and $32.7 \%$ of AR patients (skin prick tests) also had OSA diagnosed by PSG, respectively.

The fact that the prevalence of high risk patients for OSA in AR was lower than the rates reported by previous studies may be explained by the lower BMI, which an important risk factor for OSA, of our patients (mean BMI of only 23.4 $\mathrm{kg} / \mathrm{m}^{2}$ compared to 25.3 and $29.4 \mathrm{~kg} / \mathrm{m}^{2}$ in other studies). ${ }^{14,29}$ The majority of patients in this study and previous studies were female, which is the gender that has lower risk for OSA. ${ }^{30,31}$ Although PSG is accepted as a standard test for OSA diagnosis, its limitations, such as a long waiting list, a need of laborious procedures, and expensive cost, often make it impractical for most patients in a routine allergy clinic. Alternatively, in this study, we used STOP-Bang questionnaire, ${ }^{19,20,23}$ which is a much more rapid, simple, and less expensive diagnostic tool to screen for patients at high risk of being OSA.

According to the ARIA guideline, half of patients at our allergy clinic had moderate to severe nasal symptoms. However, there was no significant difference among the severity of AR classified by ARIA guideline and being high risk for OSA in this study $(p=0.60)$, and this finding corresponds with that reported by Zheng, et al. ${ }^{26}$ There was also no difference between the high and low risk OSA groups relative to the severity of each nasal symptom as assessed by VAS, which corresponds with the results reported by Bousquet, et al. ${ }^{25}$ Nevertheless, the duration of AR symptoms (sneezing, runny nose, itching, and/or nasal obstruction) was significantly longer in those with high risk of OSA $(p=0.01)$, and patients with moderate to severe AR tended to be at higher risk for having OSA than patients in the mild AR group. These findings are not surprising, possibly because the nose is known as simply a modulating factor for multilevel pharyngeal obstruction, and not a major site of upper airway collapse in OSA patients. ${ }^{5,9,10,12-16}$

\section{Limitations}

This study has some mentionable limitations. First, we used the STOP-Bang questionnaire, not PSG, which is considered the gold standard test for OSA. However, using this questionnaire is more practical and feasible than using PSG. Second, our questions regarding the duration of AR symptoms may be not specific to nasal obstruction and to the impact of treatments on patients; thus, some related biases may exist. Finally, we did not collect the data regarding the family history of atopy and current treatment as part of this study. However, we tried to reduce the effect of these confounding factors by assessing the severity of nasal symptoms only before initial treatment. The strength of this study is that this is the first report of the prevalence of OSA in South-East Asian patients with AR. These findings will increase awareness of the importance of OSA screening with questionnaires among patients in the allergy clinic setting. Early detection of high-risk OSA in AR may lead to further investigations and early treatment to prevent serious consequences of these conditions. Long-term cohort study is warranted to further elucidate the relationship between AR and OSA.

\section{Conclusion}

The prevalence of patients at high risk for OSA among patients with AR was $23.3 \%$, which is double the rate in general Thai population; however, no significant differences were observed between various severities of $\mathrm{AR}$ and being at low risk or high risk for OSA. Early detection and management of AR patients at high risk for OSA will reduce the likelihood of adverse consequences arising from either condition. 


\section{Conflict of Interest}

All authors declare no conflicts of interest relating to any aspect of this study.

\section{Acknowledgements}

The authors gratefully acknowledge the patients who agreed to join and participate in this study. We would also like to thank Dr. Saowalak Hunnangkul for her assistance with statistical analysis and data interpretation; the staff of the outpatient allergy clinic for assistance with data collection; and, the medical instructors of the Department of Otorhinolaryngology, Faculty of Medicine Siriraj Hospital, Mahidol University for their support of this study.

\section{Funding disclosure}

This study was funded by a grant from the Faculty of Medicine Siriraj Hospital, Mahidol University, Bangkok, Thailand.

\section{Author contributions}

- Surintorn Wongvilairat, MD (the principal investigator: PI) designed the study, collected and analyzed the data, and prepared the manuscript.

- Paraya Assanasen, MD designed the study, and reviewed and prepared the manuscript.

- Wish Banhiran, MD (the corresponding author) designed the study, interpretation of data, reviewed and prepared the manuscript.

- Pongsakorn Tantilipikorn, MD, PhD advised PI regarding the study methodology and statistical analysis.

- Chaweewan Bunnag, MD critically reviewed the manuscript. All authors approved the final manuscript.

\section{References}

1. Bunnag C, Jareoncharsri P, Tantilipikorn P, Vichyanond P, Pawankar R. Epidemiology and current status of allergic rhinitis and asthma in Thailand -- ARIA Asia-pacific workshop report. Asian Pac J Allergy Immunol. 2009;27:79-86.

2. Wong GW, Leung TF, Ko FW. Changing prevalence of allergic diseases in the Asia-pacific region. Allergy Asthma Immunol Res. 2013;5:251-7.

3. Canova CR, Downs SH, Knoblauch A, Andersson M, Tamm M, Leuppi JD. Increased prevalence of perennial allergic rhinitis in patients with obstructive sleep apnea. Respiration. 2004;71:138-43.

4. Ferguson BJ. Influences of allergic rhinitis on sleep. Otolaryngol Head Neck Surg. 2004;130:617-29.

5. Chirakalwasan N, Ruxrungtham K. The linkage of allergic rhinitis and obstructive sleep apnea. Asian Pac J Allergy Immunol. 2014;32:276-86.

6. Mirrakhimov AE, Sooronbaev T, Mirrakhimov EM. Prevalence of obstructive sleep apnea in Asian adults: a systematic review of the literature. BMC Pulm Med. 2013;13:10.

7. Neruntarat C, Chantapant S. Prevalence of sleep apnea in $\mathrm{HRH}$ Princess Maha Chakri Srinthorn Medical Center, Thailand. Sleep Breath. 2011;15:641-8.

8. Dorasamy P. Obstructive sleep apnea and cardiovascular risk. Ther Clin Risk Manag. 2007;3:1105-11.
9. Georgalas C. The role of the nose in snoring and obstructive sleep apnoea: an update. Eur Arch Otorhinolaryngol. 2011;268:1365-73.

10. Kohler M, Bloch KE, Stradling JR. The role of the nose in the pathogenesis of obstructive sleep apnea. Curr Opin Otolaryngol Head Neck Surg. 2009;17:33-7.

11. Kramer MF, De La Chaux R, Dreher A, Pfrogner E, Rasp G. Allergic rhinitis does not constitute a risk factor for obstructive sleep apnea syndrome. Acta Otolaryngol. 2001;121:494-9.

12. Cao Y, Wu S, Zhang L, Yang Y, Cao S, Li Q. Association of allergic rhinitis with obstructive sleep apnea: A meta-analysis. Medicine. 2018; 97:e13783.

13. Friedman M, Tanyeri H, Lim JW, Landsberg R, Vaidyanathan K, Caldarelli D. Effect of improved nasal breathing on obstructive sleep apnea. Otolaryngol Head Neck Surg. 2000;122:71-4.

14. Kalpaklioglu AF, Kavut AB, Ekici M. Allergic and nonallergic rhinitis: the threat for obstructive sleep apnea. Ann Allergy Asthma Immunol. 2009; 103:20-5.

15. Lunn M, Craig T. Rhinitis and sleep. Sleep Med Rev. 2011;15:293-9.

16. Pevernagie DA, De Meyer MM, Claeys S. Sleep, breathing and the nose. Sleep Med Rev. 2005;9:437-51.

17. Assanasen P, Banhiran W, Kositchaiwat N, Bunnag C. Prevalence of chronic rhinitis in Thai patients with obstructive sleep disordered breathing. J Med Assoc Thai. 2013;96:1169-74.

18. Senaratna CV, Perret JL, Lodge CJ, Lowe AJ, Campbell BE, Matheson MC, et al. Prevalence of obstructive sleep apnea in the general population: A systematic review. Sleep Med Rev. 2017;34:70-81.

19. Chung F, Abdullah HR, Liao P. STOP-Bang Questionnaire: A practical approach to screen for obstructive sleep apnea. Chest. 2016;149:631-8.

20. Chung F, Subramanyam R, Liao P, Sasaki E, Shapiro C, Sun Y. High STOP-Bang score indicates a high probability of obstructive sleep apnoea. Br J Anaesth. 2012;108:768-75.

21. Silva GE, Vana KD, Goodwin JL, Sherrill DL, Quan SF. Identification of patients with sleep disordered breathing: comparing the four-variable screening tool, STOP, STOP-Bang, and Epworth Sleepiness Scales. J Clin Sleep Med. 2011;7:467-72.

22. Vana KD, Silva GE, Goldberg R. Predictive abilities of the STOP-Bang and Epworth Sleepiness Scale in identifying sleep clinic patients at high risk for obstructive sleep apnea. Res Nurs Health. 2013;36:84-94.

23. Banhiran W, Durongphan A, Saleesing C, Chongkolwatana C. Diagnostic properties of the STOP-Bang and its modified version in screening for obstructive sleep apnea in Thai patients. J Med Assoc Thai. 2014;97: 644-54.

24. Brozek JL, Bousquet J, Baena-Cagnani CE, Bonini S, Canonica GW, Casale TB, et al. Allergic Rhinitis and its Impact on Asthma (ARIA) guidelines: 2010 revision. J Allergy Clin Immunol. 2010;126:466-76.

25. Bousquet PJ, Combescure C, Neukirch F, Klossek JM, Mechin H, Daures JP, et al. Visual analog scales can assess the severity of rhinitis graded according to ARIA guidelines. Allergy. 2007;62:367-72.

26. Zheng M, Wang X, Ge S, Gu Y, Ding X, Zhang Y, et al. Allergic and non-allergic rhinitis are common in obstructive sleep apnea but not associated with disease severity. J Clin Sleep Med. 2017;13:959-66.

27. Young T, Finn L, Kim H. Nasal obstruction as a risk factor for sleep-disordered breathing. J Allergy Clin Immunol. 1997;99:757-62.

28. Stuck BA, Czajkowski J, Hagner AE, Klimek L, Verse T, Hormann K, et al. Changes in daytime sleepiness, quality of life, and objective sleep patterns in seasonal allergic rhinitis: a controlled clinical trial. J Allergy Clin Immunol. 2004;113:663-8.

29. Bozkurt B, Serife Ugur K, Karamanli H, Kucuker F, Ozol D. Polysomnographic findings in persistent allergic rhinitis. Sleep Breath. 2017;21:255-61.

30. Al Lawati NM, Patel SR, Ayas NT. Epidemiology, risk factors, and consequences of obstructive sleep apnea and short sleep duration. Prog Cardiovasc Dis. 2009;51:285-93.

31. Banhiran W, Junlapan A, Assanasen P, Chongkolwatana C. Physical predictors for moderate to severe obstructive sleep apnea in snoring patients. Sleep Breath. 2014;18:151-8. 